\title{
Three Staged Revision Arthroplasty Using Structural Allograft in Chronic Periprosthetic Joint Infection Accompanied by Fracture: A Case Report
}

\section{Sai-Won Kwon}

Department of Orthopaedic Surgery, Soonchunhyang University Cheonan Hospital, Soonchunhyang University College of Medicine, Cheonan, Korea

\begin{abstract}
Revision arthroplasty for chronic periprosthetic joint infection is complex and determined by many variables. Generally, two-staged revision arthroplasty is the standard treatment for the management of chronic periprosthetic joint infection. However, it is difficult to resolve chronic infection accompanied by large bone deficiency due to pathologic fracture. I report a case of successful threestaged revision arthroplasty using frozen structural allograft in chronic periprosthetic knee joint infection accompanied by extensive bone defect due to fracture.
\end{abstract}

Keywords: Persistent infection; Periprosthetic fractures; Bone defect; Structural allograft; Revision arthroplasty; Case report

\section{INTRODUCTION}

Periprosthetic joint infection (PJI) is the most difficult and challenging complication. Despite significant effort, the incidence of PJI in primary total knee arthroplasty (TKA) ranges from $0.4 \%$ to $2.0 \%$ [1]. Revision arthroplasty for chronic PJI is very difficult and determined by many variables. Generally, two-staged revision arthroplasty combined with appropriate antibiotic therapy is the standard treatment for the management of chronic PJI [2]. Chronic PJI increases the risk of periprosthetic fracture by lytic changes the bony architecture during the progress, suggesting the need for surgical readiness. Large periprosthetic bone deficiency requires multiple management strategies involving revision total knee arthroplasty and distal femoral structural allograft.

\section{CASE REPORT}

An 80-year-old woman underwent TKA for degenerative osteoarthritis of right knee 17 months ago at a local clinic. She presented at our outpatient department with symptoms of pain, swelling, heating, and limited motion of the operated knee. Laboratory evaluation carried out in an outpatient setting revealed an increase in C-reactive protein (CRP) level up to $2.35 \mathrm{mg} / \mathrm{dL}$ (normal $<0.5 \mathrm{mg} / \mathrm{dL}$ ) and an elevation in erythrocyte sedimentation rate (ESR) of $114 \mathrm{~mm} / \mathrm{hr}$ (normal $<30 \mathrm{~mm} / \mathrm{hr}$ ). The polymorphonuclear cell distribution in the joint fluid was $91 \%$. Methicillin sensitive staphylococcus aureus (MSSA) was cultured in the sample. Initial plain radiography revealed periprosthetic osteolysis in distal femur without evidence of fracture (Fig. 1A). The patient was diagnosed with chronic PJI by MSSA based on clinical and laboratory investigations. A twostaged revision arthroplasty was planned, but the patient fell while hospitalized. A pathologic fracture was found on a plain radiograph taken after admission (Fig. 1B).

The first stage surgery was performed via a previous medial parapatellar approach. The implant was removed easily. The distal fragment of the fracture was increasingly unstable due to large bone defects involving both distal femur and proximal tibia (Anderson Orthopedic Research Institute [AORI] classification type IIB) [3] (Fig. 2A). After removal of whole implants and appropriate debridement, AORI classification was converted to type III. The large fracture fragment was fixed by multiple Kirschner wires to ensure temporary stability (Fig. 2B). Subsequently, the vancomycin-impregnated polymethylmethacrylate (VPMMA) beads and VPMMA spacer were inserted between the joint space (Fig. 2C). First-generation cephalosporin was applied to control the infection for 8 weeks. The callus of distal femoral bone fragment didn't 

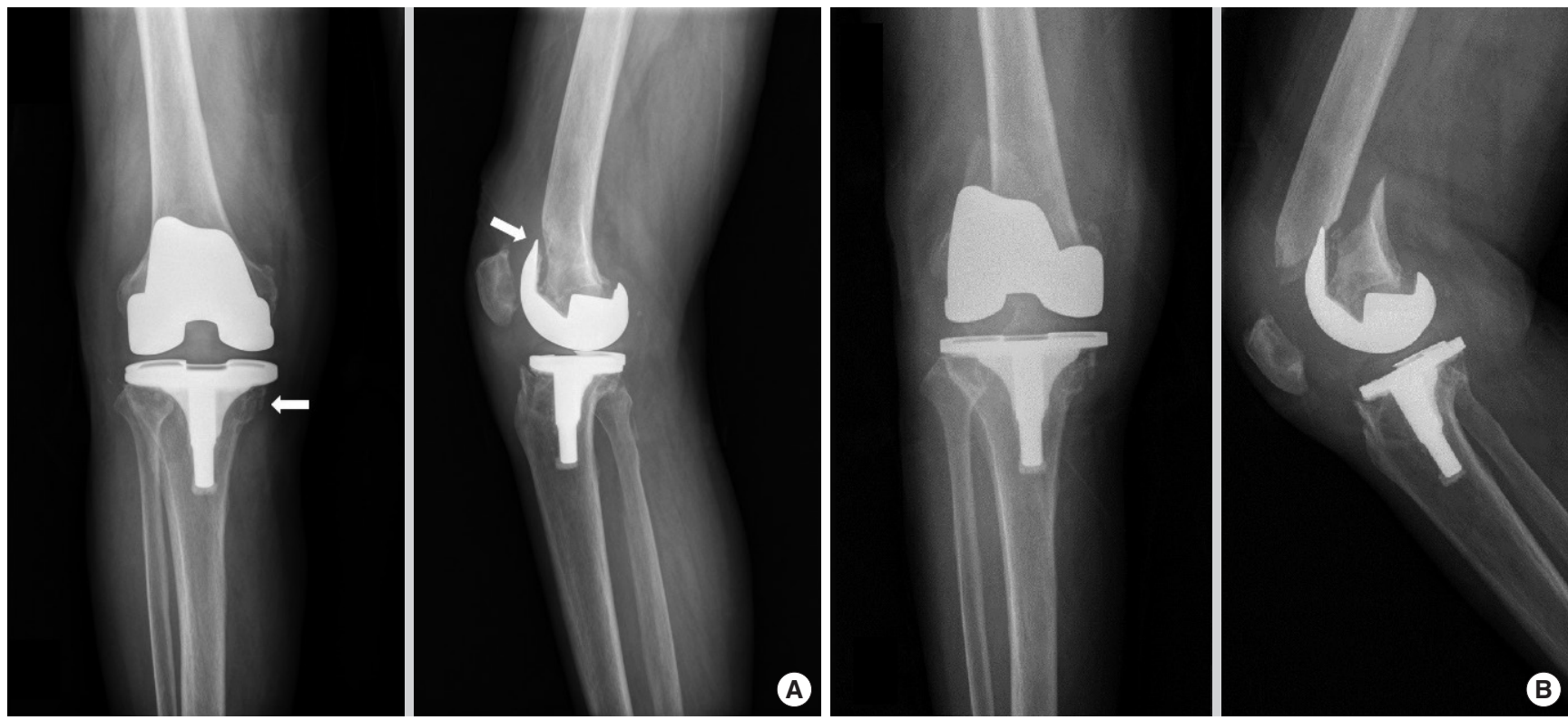

Fig. 1. Preoperative radiographs of the right knee demonstrated osteolysis around metal implants (white arrows) (A) and shows periprosthetic fracture at supracondylar level (B).
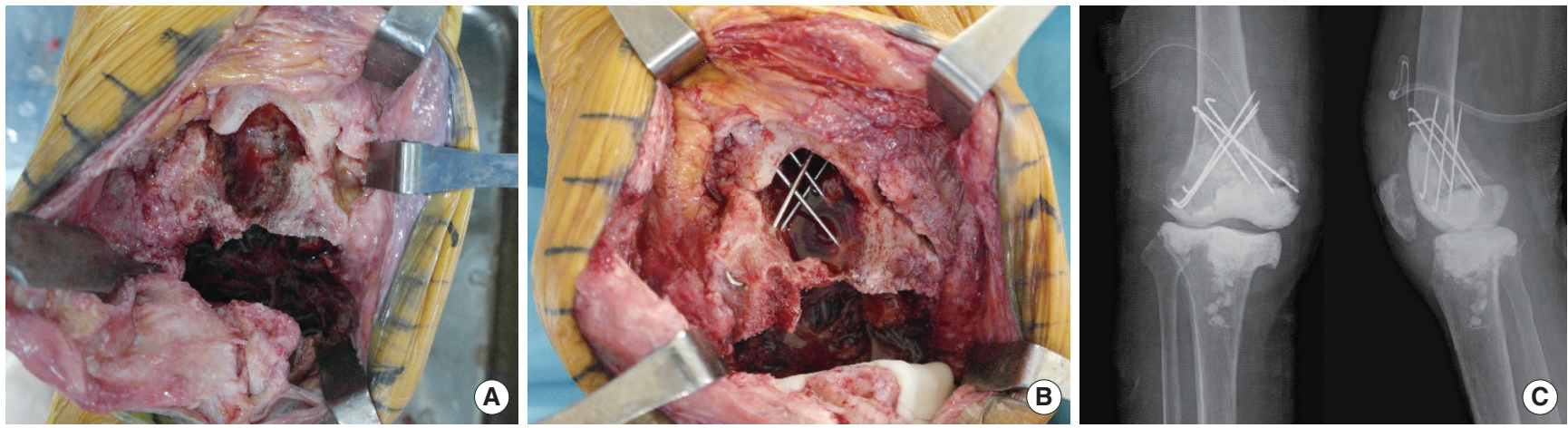

Fig. 2. Photograph (A) shows large cavitary femoral metaphyseal bone loss and fracture after total prosthesis removal. Photograph (B) and radiograph (C) show the postoperative joint condition secured by multiple Kirschner wires and covered with vancomycin-impregnated polymethylmethacrylate spacers.

be shown and fracture had angulation on plain radiograph (Fig. 3); however, laboratory markers such as CRP and ESR were normalized after 4 weeks of antibiotic treatment.

Eight weeks after the first surgery, the second stage revision surgery was performed. The VPMMA-bead, VPMMA spacer and Kwire, which were inserted in a previous surgery, were removed. In the absence of signs of active inflammation, the distal bone fragment was removed except for both heads of gastrocnemius muscle, because the fragment of distal femur was considered non-viable. The Frozen allo-bone graft (right distal femur, $18 \mathrm{~cm}$; Korea Public Tissue Bank, Seoul, Korea) for structural replacement was used to manage the large defect according to the size and length of the

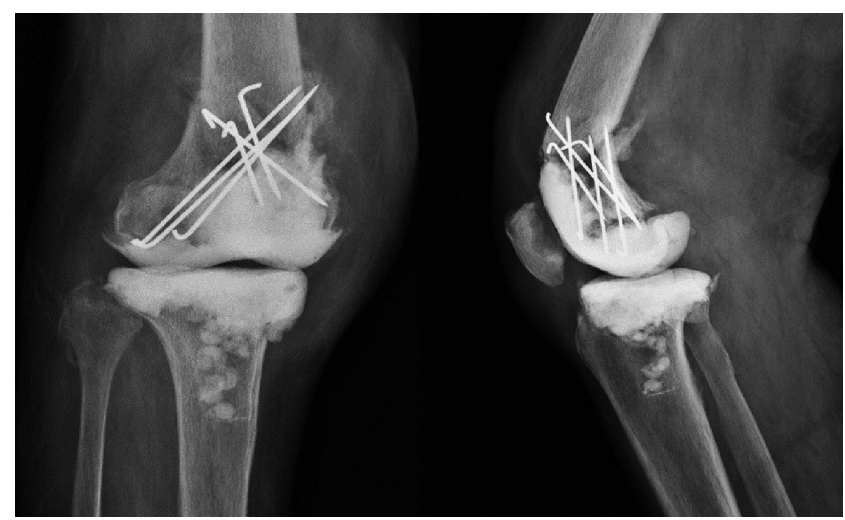

Fig. 3. Eight weeks after the first stage surgery, plain radiographs showed no callus around the fracture and only angular deformity. 

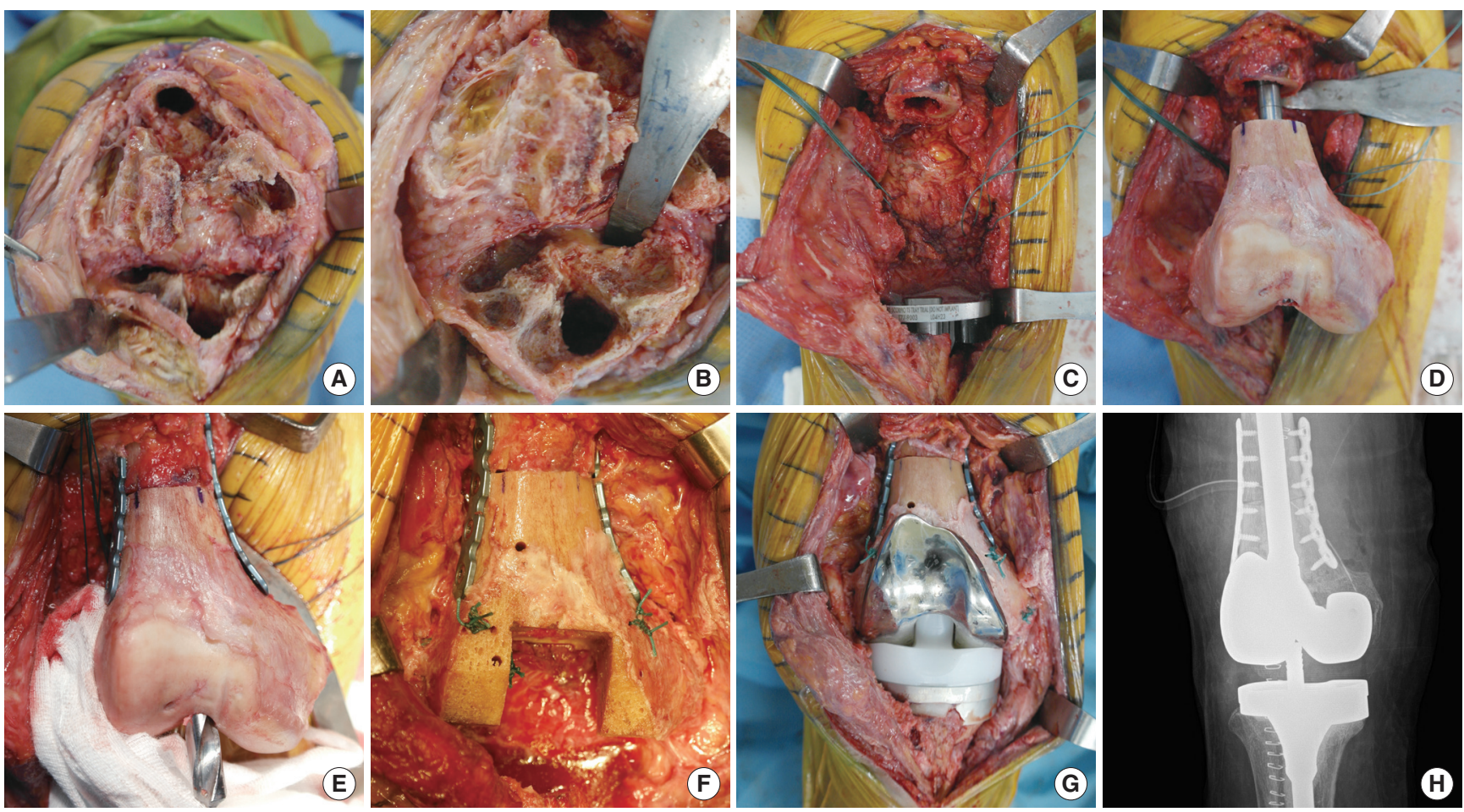

Fig. 4. (A-H) Photographs show the process of the second stage surgery by removing the dead bone fragments and transplanting structural allografts to perform joint replacement arthroplasty.

opposite distal femur. First, the femoral reamer was inserted into intramedullary cavity of the box cut structural allograft to correct rotation and axis. The allograft was fixed firmly with a dual plate (Arix locking plate 6H; JEIL medical, Seoul, Korea) followed by revision arthroplasty using semi-constrained typed devices (Scorpio TS; Stryker, Kalamazoo, MI, USA). And then the two heads of gastrocnemius muscle were firmly fixed to their original anatomical location on the femoral allograft using a pull-out technique (Fig. 4). The long leg cast brace was applied for postoperative stability. After 3 days of surgery, continuously passive motion (CPM) exercise was started and gradually increased to $90^{\circ}$ flexion. The patient began toe-touched partial weight-bearing ambulation while wearing the brace. Stable implant placement and plate fixation were observed on plain radiographs 6 weeks after surgery. However, multidirectional instability remained.

In step 3, 8 weeks after the second operation, reconstruction of the posterolateral corner and medial collateral ligament was performed using an Achilles tendon allograft with bone fragment attached. Reconstruction was performed with bilateral incision, especially the posterolateral corner reconstruction used by the modified Larson technique (Fig. 5). The operated knee joint was pro-
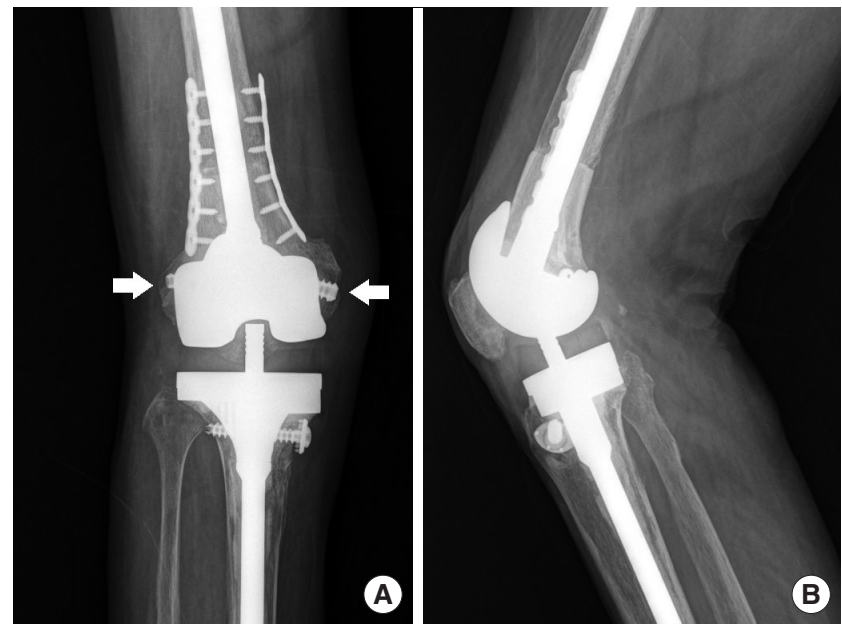

Fig. 5. (A, B) Plain radiographs show the interference screws (white arrows) inserted after bilateral collateral ligament reconstruction in the third stage surgery.

tected with a long leg cast immobilization for 4 weeks postoperatively and replaced with the brace. CPM and stationary bicycling exercises were performed with the applied braces. Three months after the third stage surgery, a full active range of motion was obtained and simple radiographs showed a stable knee joint. At a 
year follow-up, the patient had no pain after the third surgery and showed stable joint movement on physical examination. Bone union was observed between the patient's femur shaft and the grafted allograft.

\section{DISCUSSION}

A variety of treatment options in infection after TKA are available, including long-term antibiotic therapy, open debridement, resection arthroplasty, one- or two-stage revision arthroplasty, and arthrodesis. Among them, two-staged revision arthroplasty combined with appropriate antibiotic therapy has been described by many researchers $[1,2,4]$. Romano et al. [4] reviewed that the average success rate was $81.9 \%$ for one-stage surgery and $89.8 \%$ for two-stage surgery in meta-analysis. Therefore, two-stage revision arthroplasty in widely used as an appropriate treatment method for chronic PJIs. As in this case, if a periprosthetic fracture occurs and the bone deficiency becomes larger than expected in the process of considering two-stage revision surgery, additional solutions to secure structural stability to facilitate revision surgery are necessary in addition to controlling the infection. Revision total knee arthroplasty is associated with potential challenges due to the varying nature of bone loss. Management of bone loss depends on the location and size of the defect, and variable factors including age, activity, and life expectancy. In general, small contained defects (AORI type I) are treated with cement filling [5]. For larger defects (AORI type II), modular augmentation or bone grafts are suitable. Massive bone loss (AORI type III) is difficult to treat, although there are several management options. The porous metal cone augments may be used for management of large metaphyseal defects [6]. Frozen structural allograft can be used to replace extensively lost bone, although it is associated with the risk of infection and long-term fusion with host bone. Stockley et al. [7] reported the use of structural grafts to reconstruct defects around the distal femur. Step-cut osteotomy was adopted at the allografthost bone junction to ensure primary stability without the need for plates during bulk allograft fixation. However, in this case, the bone stock was not adequate for step-cut osteotomy and fixation with multiple wires to the host bone. Transverse osteotomy was performed and fixed the allograft-host bone with bilateral plates to calibrate rotation and ensure stable fixation.

In addition to structural stability, proper soft-tissue balancing and sufficient ligamentous stability are important for successful revision TKA [8]. In revision TKA, damage to the ligament structure was generally attributed to loss of the femoral epicondylar bone leading to instability. MCL reconstruction during primary TKA was mentioned in a few studies; however, PLC reconstruction during revision arthroplasty is very rare. Peters et al. [9] reported MCL reconstruction in primary TKA and Ohnsorge et al. [10] reported PLC reconstruction in revision knee arthroplasty. However, in this case, since sufficient bone base for ligament fixation was not secured, bilateral collateral ligament reconstruction was performed in stages. Ligament reconstruction was performed using calcaneus Achilles allograft or bone patellar tendon allograft to obtain firm bone fixation [9].

In conclusion, revision arthroplasty for chronic infection associated with extensive bone loss is a challenging task for orthopedic surgeons. Proper management of bone loss for stable fixation and accurate balancing of ligaments is essential for successful revision arthroplasty. Revision arthroplasty using structural bone graft and ligament reconstruction could be one of the optional salvage procedures when simple adjustment or repair is not possible.

\section{CONFLICT OF INTEREST}

No potential conflict of interest relevant to this article was reported.

\section{REFERENCES}

1. Kurtz SM, Ong KL, Lau E, Bozic KJ, Berry D, Parvizi J. Prosthetic joint infection risk after TKA in the Medicare population. Clin Orthop Relat Res 2010;468:52-6.

2. Hirakawa K, Stulberg BN, Wilde AH, Bauer TW, Secic M. Results of 2-stage reimplantation for infected total knee arthroplasty. J Arthroplasty 1998; 13:22-8.

3. Engh GA, Herzwurm PJ, Parks NL. Treatment of major defects of bone with bulk allografts and stemmed components during total knee arthroplasty. J Bone Joint Surg Am 1997;79:1030-9.

4. Romano CL, Gala L, Logoluso N, Romano D, Drago L. Two-stage revision of septic knee prosthesis with articulating knee spacers yields better infection eradication rate than one-stage or two-stage revision with static spacers. Knee Surg Sports Traumatol Arthrosc 2012;20:2445-53.

5. Radnay CS, Scuderi GR. Management of bone loss: augments, cones, offset stems. Clin Orthop Relat Res 2006;446:83-92.

6. Richards CJ, Garbuz DS, Pugh L, Masri BA. Revision total knee arthroplasty: clinical outcome comparison with and without the use of femoral head structural allograft. J Arthroplasty 2011;26:1299-304.

7. Stockley I, McAuley JP, Gross AE. Allograft reconstruction in total knee arthroplasty. J Bone Joint Surg Br 1992;74:393-7.

8. Kelly MA. Ligament instability in total knee arthroplasty. Instr Course Lect 2001;50:399-401. 
9. Peters CL, Dienst M, Erickson J. Reconstruction of the medial femoral condyle and medial collateral ligament in total knee arthroplasty using tendoachilles allograft with a calcaneal bone block. J Arthroplasty 2004; 19:935-40.
10. Ohnsorge JA, Wickiewicz TL, Davis J, Laskin RS. Revision knee arthroplasty including reconstruction of the lateral collateral ligament by allograft: a case report. HSS J 2010;6:219-22. 